\title{
Biotechnology-A Horticulturist's Perspective: Introduction to the Colloquium
}

\author{
Stanley J. Kays \\ Department of Horticulture, University of Georgia, Athens, GA 30602
}

\begin{abstract}
The colloquium "Biotechnology: Implications for Improved Quality in Horticultural Products" was developed by the Postharvest Working Group and cosponsored by the Genetics and Germplasm, Fruit Breeding, and Vegetable Breeding Working Groups of ASHS. Our collective interest in the colloquium is based upon what we see as the potentially profound impact of the use of recombinant DNA technology in the development of crop plants, The use of recombinant DNA techniques in genetic engineering represents one segment of biotechnology-the one largely focused on in this colloquium. In the very immediate future, this technology will alter each of our professional programs-whether we are in extension, teaching, or research with an educational institution, in industry, or in a government laboratory.
\end{abstract}

One often hears the view that plants engineered by recombinant DNA technology are a thing of the future. This is no longer the case. As the following reports will indicate, the future is now. Since the first transgenic plants were reported in 1983, we now have plants that have been genetically engineered for resistance to herbicides, insects, and viruses. The use of recombinant DNA in plant genetic engineering is here. It is starting to have what will prove to be a phenomenal impact on our lives.

Major technological advances seldom cause instantaneous change in a society. Rather, advances typically proceed through several stages. Initially, a new technology is used to accomplish what has already been done, only more effectively or efficiently. This is followed by applying the technology to new uses. Eventually, the technology begins to alter the very society in which it is used.

If one looks at the major technological innovations in the last century, advances such as the internal combustion engine and the computer, the collective impact of these inventions has been tremendous. However, when contrasted with the potential impact on our society of the use of recombinant DNA technology, the latter promises to be infinitely greater. Because of this, it is essential that each of us, as horticulturists, has a clear impression of what genetic engineering is, the existing state of the art, and how it may modify and shape each of our individual programs.

From a historical perspective, the use of recombinant DNA techniques is the next logical step in the modification of plants by man for his use, About 10,000 years ago, our ancestors began to cultivate the seeds of several wild grain species. Superior progeny were selected and used, thus beginning man's modification and domestication of plants. The development of agriculture had a monumental impact on the structure of civilization and opened the door for all of the complex societies and civilizations that followed.

The next major step was the widespread use of plant breeding. Plants with beneficial traits were pragmatically crossed and the progeny that proved to be superior to the parents selected for production of the next generation. Plant breeders have had a tremendous impact on the availability of food and fiber for man. In the past 50 years, the yields of the major grain crops have doubled and, in some cases, tripled. Comparable achievements have been reported for many horticultural crops.

Despite these successes, plant breeders have been limited in what they can achieve and how rapidly progress can be made. Breeding is a time-consuming practice. Depending on the crop, it may require years before an improved cultivar can be released, because conventional breeding combines the entire genomes of both parents. The result is a myriad of possible combinations that must be prag- 
matically examined to find the best. Additional crosses or backcrosses with one of the parents are often required, adding time to the process. Likewise, conventional breeding is limited to a relatively narrow genetic base since the trait must be present in a sexually compatible species. Even within a species, there is often considerable incompatibility, impeding the incorporation of selected traits into new cultivars. The use of recombinant DNA opens the door to a vast array of beneficial genes-genes that are not restricted to a given plant species, but may be obtained from any organism.

The ability to isolate, clone, and insert specific genes into plants allows specific improvements in superior lines. At present, however, only traits controlled by one or very few genes can be transferred. Likewise, our knowledge of the mechanisms controlling gene expression is limited. Insertion of a gene that is transcribed in the wrong tissue, at the wrong time, or to an undesired degree is of little value.

Two approaches that have been proposed for the incorporation of superior genes using recombinant DNA are: 1) insertion in a superior line from a conventional breeding program; or 2) insertion in a sexually compatible line that then can be used as a parent in a conventional breeding program. With either, it is evident that gene transfer using recombinant DNA technology will not replace conventional breeding programs. Rather, it represents a powerful new tool for plant breeders that will allow them to achieve previously unthinkable accomplishments with their individual crops.

Since the number of horticulturists using recombinant DNA represents only a minute percentage of our total Society, our intent was to present a colloquium directed toward horticulturists not involved in recombinant DNA research. In the following reports, we hope to provide: 1) an overview of how recombinant DNA is used to produce transgenic plants; 2) an understandable critique of the new vocabulary that has emerged; 3 ) the state of genetic engineering for horticultural crops; 4) a description of some of the problems that are slowing progress; and 5) the potential for the use of recombinant DNA in horticulture. 